\title{
Identité culturelle et scolarité d'élèves portugais en Suisse
}

\section{Francisco Pons, Pierre-André Doudin et Gianreto Pini}

Dans cet article, nous étudions la relation entre l'identité culturelle et certaines caractéristiques personnelles, familiales et scolaires d'élèves migrants d'origine portugaise achevant leur scolarité obligatoire en Suisse (canton de Vaud). L'identité culturelle est mesurée au moyen d'une adaptation de l'échelle d'acculturation de Berry. Les résultats montrent que ces élèves ont tendance, d'une part, à vouloir très clairement préserver leur culture d'origine tout en intégrant en même temps la culture suisse (attitude clairement pro-intégration) et, d'autre part, sont contre un rejet de la culture suisse (attitude clairement anti-séparation) et portugaise (attitude légèrement antiassimilation). Cependant, en fonction de certaines caractéristiques personnelles (sexe, date d'arrivée en Suisse), familiales (type de permis de travail de la mère) et scolaires (redoublement, niveau de réussite scolaire, fréquentation des cours de langue et de civilisation portugaises), nous constatons des fléchissements significatifs de certaines de ces attitudes et notamment de l'attitude pro-intégration. Ces résultats sont discutés du point de vue de leurs implications sur le plan scolaire (danger de marginalisation scolaire) et sur le plan politique (danger de marginalisation sociale).

\section{Problématique}

Dans cet article, nous présentons les résultats d'une recherche portant sur la relation entre l'intégration scolaire et l'identité culturelle d'élèves migrants d'origine portugaise vivant en Suisse (canton de Vaud). Plus précisément, nous analysons le lien entre certaines caractéristiques des élèves, de leur famille et de leur cursus scolaire d'une part et leur mode d'acculturation d'autre part.

Le Portugal a une longue tradition d'immigration au point que Silva et Neto (1993) y voient une constante structurale de la société portugaise. Près d'un tiers des Portugais résident hors de leur pays. Plusieurs pays (France, Luxembourg, Suisse, Canada, Australie, etc.) ont eu un important flux migratoire d'origine portugaise. Ainsi en Suisse romande, le portugais constitue la deuxième langue maternelle la plus parlée après le français par les élèves de la scolarité obligatoire 
(OFS, 1995). Malgré cet état de fait, les recherches portant sur les élèves migrants portugais sont relativement peu nombreuses (Arroteia et Doudin, 1998, pour une revue récente). Elles peuvent être regroupées en trois courants selon qu'elles s'intéressent à leur scolarité, à leur identité culturelle ou au lien entre ces deux réalités.

Tout d'abord, des études montrent que le taux de réussite scolaire des élèves portugais expatriés est généralement inférieur à celui des adolescents français (Bastide, 1994; Neto, 1993), canadiens ou australiens (Berry, Klim, Power et Young, 1989) et suisses (Doudin, 1996a). En ce qui concerne ce dernier pays, Doudin (1998), Doudin, Pons et Moreau (1998) montrent que la qualité de l'intégration scolaire ne dépend pas des aides fournies par l'école tant à l'arrivée du jeune migrant (classes d'accueil) qu'au cours de sa scolarité (appui pédagogique, redoublement, classe regroupant des élèves en difficulté), celles-ci ayant plutôt un effet négatif. Cet effet négatif peut s'expliquer par l'aspect paradoxal des aides qui consiste à vouloir mieux intégrer l'élève sur le plan scolaire tout en l'excluant - de son groupe d'âge en le faisant redoubler - de la classe régulière, soit momentanément (appui), soit durablement (classe d'accueil), voire, dans certains cas, définitivement (classe regroupant des élèves en difficulté). Ce paradoxe peut produire des effets d'autant plus négatifs que ces formes d'exclusion touchent justement des élèves migrants qui tentent de sintégrer à leur pays d'accueil. Par contre, la qualité de l'intégration scolaire dépend de caractéristiques familiales, comme le niveau d'éducation des parents et le type de permis de travail ${ }^{1}$ obtenu par les parents à leur arrivée en Suisse. Ainsi par exemple, le fait que le père et/ou la mère ait été détenteur d'un permis de saisonnier (permis A) a des effets très négatifs sur la scolarité de l'enfant. De par l'interdiction qui est faite à la famille de rejoindre en Suisse le détenteur de ce type de permis, il s'agit d'une attaque contre la cohésion familiale et les fonctions parentales ne sont plus assumées de manière optimale. Les résultats de Cattafi-Maurer et al. (1998) vont dans le même sens. La précarité juridique des jeunes portugais et celle de leurs parents affecterait notamment leur intégration scolaire et leur accès à une formation professionnelle.

Ensuite, des études ont investigué le problème de l'identité culturelle. Ainsi, les Portugais expatriés choisissent en général un compromis entre les valeurs de la culture portugaise et celles du pays d'accueil, que ce soit en France (Neto, 1994, 1995), au Canada ou en Australie (Berry, Klim, Power et Young, 1989; Lanca, Alksinis, Roese et Gardner, 1994). L'identité culturelle évolue en fonction de la durée du séjour dans le pays d'accueil. Comme le montrent Berry et al. (1989), chez des adultes, plus la durée du séjour est longue, plus ils ont tendance à abandonner l'identité culturelle du pays d'origine en faveur de celle du pays d'accueil. Mais l'identité culturelle varie également en fonction du contexte ou de la politique menée par le groupe dominant du pays d'accueil vis-à-vis des groupes minoritaires comme les migrants. Par exemple, les Portugais vivant dans la zone anglophone du Canada s'identifient plus à la culture nord-américaine 
que ceux vivant dans la zone francophone à la culture québécoise (Lanca et al., 1994).

Enfin, les investigations sur la relation entre l'identité culturelle et des variables cognitives sont rares (van de Vijver, Helms-Lorenz et Feltzer, 1999, pour une revue récente), voire quasi inexistantes et anciennes lorsque ces variables sont de nature scolaire (Baldauf et Ayabe, 1977; Inkeles et Smith, 1974). La recherche menée en France par Bastide (1994) montre que l'identité culturelle entretient un lien étroit avec la qualité de l'intégration scolaire: en effet, l'échec scolaire est plus fréquent chez les élèves qui se réclament d'une identité portugaise que chez ceux qui se réclament d'une double identité portugaise et française ou d'une identité plutôt française.

Aussi, dans le cadre d'une recherche sur les difficultés d'intégration scolaire d'adolescents portugais (Doudin, 1998), il nous a paru intéressant d'investiguer également l'identité culturelle de ces jeunes et d'aller plus avant dans l'analyse de la relation entre la scolarité et l'identité culturelle. C'est l'objectif de ce travail que de présenter les résultats de cette recherche. Afin d'analyser cette relation, il est nécessaire de choisir, d'une part, des indicateurs rendant compte de la scolarité des élèves et, d'autre part, un modèle rendant compte de leur identité culturelle. Nous présentons ci-après les choix opérés.

\section{Indicateurs scolaires}

Comme le définit Nuttall (1992, p. 17), «les indicateurs ont pour objet de fournir un aperçu du comportement et de l'efficacité des systèmes d'enseignement...». Les recherches sur les indicateurs scolaires ont pris beaucoup d'ampleur ces dernières années dans de nombreux pays (par ex. OCDE, 1996). Les autorités scolaires, les chercheurs en éducation ainsi que les enseignants s'intéressent de plus en plus aux performances du système éducatif avec, pour objectif, d'améliorer le niveau de formation des élèves en général et plus particulièrement des élèves appartenant à des minorités ethniques ou à des groupes socio-économiques défavorisés. Il existe de nombreuses façons de catégoriser les indicateurs. Par exemple, Bottani et Walberg (1992) proposent de classer les indicateurs de l'enseignement en quatre types:

1. les indicateurs d'input; caractéristiques des élèves lorsqu'ils entrent dans le système éducatif (sexe, niveau socio-économique de sa famille, etc.);

2. les indicateurs de processus; caractéristiques de l'organisation de l'école (programmes, pratiques pédagogiques, mesures de pédagogie compensatoire, etc.);

3. les indicateurs d'output; caractéristiques des élèves lorsqu'ils sortent du système éducatif (type d'enclassement final, taux d'abandon, etc.);

4. les indicateurs de ressources financières et humaines (budgets, effectifs des enseignants, formation des enseignants, etc.).

Le choix des indicateurs doit bien entendu refléter les caractéristiques principales de la population d'élèves étudiés (y compris les caractéristiques de leur famille) et du système scolaire dont ils sont issus. Afin de procéder à ce choix, nous nous ré- 
férons d'une part aux résultats de la première partie de notre recherche (Doudin, Pons et Moreau, 1998) et, d'autre part, à l'analyse du système scolaire vaudois (Doudin, 1996a).

Au tableau 1, nous récapitulons les indicateurs scolaires retenus en fonction de leur type. Nous pouvons remarquer que l'analyse porte sur trois des quatre catégories d'indicateurs, les indicateurs de ressources financières et humaines ne faisant pas partie de l'analyse.

Tableau 1: Indicateurs scolaires retenus par type

\begin{tabular}{|l|l|}
\hline Indicateurs & Type d'indicateur \\
\hline Sexe de l'élève & input \\
Age d'arrivée de l'élève en Suisse & input \\
Niveau de formation du père/de la mère & input \\
Permis de travail du père/de la mère & input \\
\hline Redoublement & processus \\
Changement ascendant de division & processus \\
Fréquentation des cours de langue et de civilisation portugaises & processus \\
\hline Enclassement en fin de scolarité obligatoire & output \\
Projet de formation post-obligatoire & output \\
\hline
\end{tabular}

Trois indicateurs nous renseignent plus particulièrement sur le niveau général de réussite scolaire de l'élève: le redoublement (l'élève est resté au moins une fois au même niveau durant deux années scolaires), le changement ascendant de division (l'élève a changé positivement au moins une fois de division) et l'enclassement en fin de scolarité obligatoire (la division fréquentée par l'élève à la fin de sa scolarité). La combinaison de ces trois indicateurs nous permet de définir cinq types de niveau général de réussite scolaire allant du plus négatif au plus positif. Dans le premier (type «Très faible»), le sujet est au niveau d'enclassement le plus bas, il a redoublé et n'a jamais changé de division. Dans le deuxième (type «Faible»), le sujet est au niveau d'enclassement le plus bas, mais il a soit redoublé et changé de division, soit ni redoublé ni changé de division, soit pas redoublé mais changé de division. Dans le troisième (type «Moyen»), le sujet se trouve au niveau d'enclassement intermédiaire et il a redoublé, mais n'a pas changé de division. Dans le quatrième (type «Bon»), le sujet se trouve au niveau de classement intermédiaire, mais il a soit redoublé et changé, soit ni redoublé ni changé, soit pas redoublé mais changé de division. Enfin, dans le cinquième (type «Très bon»), le sujet a l'enclassement le plus élevé, il a ou n'a pas redoublé et a eu un changement ascendant de division.

\section{Identité culturelle}

La psychologie sociale et la psychologie interculturelle sont parmi les principaux courants de recherche qui ont investigué le problème de l'identité sociale. En psychologie sociale, par exemple Deschamps (1991), Turner (1987) ont étudié l'identité sociale; Kuhn et MacPartland (1954), Zavalloni et Guerin (1984), Tajfel, Biling, Bundy et Flament (1971) ont proposé divers instruments pour 
mesurer cette identité. C'est dans ce cadre, que notamment, Pérez, Mugny, Llavata et Fierres (1993) ont analysé l'identité culturelle de groupes minoritaires. En psychologie interculturelle, le modèle dominant est celui de Berry (Berry, 1989, 1995, 1999; Berry et al., 1989; Berry et Sam, 1997; Dona et Berry, 1994; Krishnan et Berry, 1992). C'est ce modèle que nous avons adopté pour cette présente étude car, à la différence des modèles psychosociaux susmentionnés, il porte directement sur l'identité culturelle des migrants. Nous le résumons brièvement.

Selon Berry, toute personne immigrée présente des modes d'acculturation qui résultent de la relation qu'elle entretient avec sa culture d'origine et la culture du pays d'accueil. L'adoption ou le rejet par un individu de l'un ou de l'autre mode d'acculturation dépend, d'une part, de l'attitude que les individus eux-mêmes ont à l'égard de l'acculturation et, d'autre part, de la politique d'acculturation du pays d'accueil, cette politique dépendant essentiellement du degré de tolérance à l'égard des différences culturelles. Autrement dit, dans les sociétés culturellement diversifiées, tous les individus présentent des attitudes relatives à leurs relations avec les différents groupes (dominants ou minoritaires) constitutifs de ces sociétés. Pour des individus dont le groupe est minoritaire, comme les Portugais en Suisse, un problème se pose: la préservation de l'identité culturelle de son groupe et les rapports avec l'identité culturelle des autres groupes, et plus particulièrement avec celle du groupe dominant, à savoir la culture suisse.

Selon Berry, il existe au moins quatre réponses différentes à ce problème, que l'on peut désigner par le terme de «modes d'acculturation». Nous décrivons brièvement ces quatre modes tout en les illustrant par un exemple issu de notre questionnaire:

1. l'assimilation consiste en l'abandon de l'identité culturelle du pays d'origine en faveur de l'identité culturelle dominante du pays d'accueil. Dans notre recherche, elle consiste en la négation des valeurs de la culture portugaise en faveur des valeurs de la culture suisse. Cette attitude peut être illustrée par l'affirmation suivante: «Parce que nous vivons en Suisse, nous n'avons pas besoin de parler la langue portugaise. Nous devrions apprendre uniquement la langue française»;

2. la séparation consiste en un rejet de l'identité culturelle dominante du pays d'accueil en faveur de la culture du pays d'origine. Dans notre recherche, elle consiste en la négation des valeurs de la culture suisse en faveur des valeurs de la culture portugaise. Cette attitude peut être illustrée par l'affirmation suivante: «Les Portugais devraient vivre ensemble pour avoir moins de contacts avec les Suisses»;

3. l'intégration consiste en un compromis entre l'identité culturelle d'origine et celle dominante du pays de résidence. Dans notre recherche, elle consiste à la fois en une reconnaissance des valeurs de la culture portugaise et des valeurs de la culture suisse. Cette attitude peut être illustrée par l'affirmation suivante: «J'encouragerai les enfants portugais élevés en Suisse à étudier l'histoire 
suisse et l'histoire portugaise, car c'est important pour eux de connaître l'histoire de ces deux pays»;

4. la marginalisation consiste en un rejet à la fois de l'identité culturelle dominante du pays d'accueil et de l'identité culturelle du pays d'origine. Dans notre recherche, elle consiste en la négation des valeurs à la fois de la culture suisse et de la culture portugaise. Cette attitude peut être illustrée par l'affirmation suivante: «Je n'aime ni la nourriture portugaise, ni la nourriture suisse parce que les deux sont mauvaises pour ma santé.»

\section{Méthode}

\section{Échantillon}

83 adolescent(e)s Portugais(es) volontaires ont été examiné(e)s. Tous les sujets suivaient leur dernière année d'école obligatoire (9e) au sein du système scolaire du canton de Vaud (Suisse Romande). Ils étaient âgés de 15 ans 3 mois à 17 ans 11 mois, 60\% étaient des filles et 40\% des garçons.

\section{Mesures}

Les sujets ont été examinés deux fois, à 6 mois d'intervalle, au moyen de deux questionnaires qu'ils devaient remplir individuellement à leur domicile. Le premier permettait d'analyser certains indicateurs scolaires d'input, de processus et d'output. Le deuxième a servi à évaluer divers aspects de leurs modes d'acculturation vis-à-vis des cultures portugaise (pays d'origine) et suisse (pays d'accueil).

Pour mesurer l'attitude du sujet vis-à-vis des quatre modes d'acculturation (assimilation, séparation, intégration, marginalisation), nous avons procédé à une adaptation de l'échelle de Berry (communication personnelle). Nous avons demandé aux sujets de se situer par rapport à 48 affirmations représentatives de chacun de ces modes (12 affirmations par mode) au moyen d'une échelle en 5 points (1- «complètement en désaccord»; 2- "plutôt en désaccord»; 3- «neutre»; 4- "plutôt d'accord»; 5- «complètement d'accord»). La moyenne des réponses aux 12 affirmations caractéristiques d'un mode d'acculturation a été ensuite calculée, ce qui a permis d'obtenir pour chaque sujet quatre moyennes représentatives de l'amplitude de ces attitudes vis-à-vis de chacun des quatre modes d'acculturation. Une moyenne élevée indique que le sujet est plutôt en accord avec les affirmations constitutives d'un mode d'acculturation; au contraire, une moyenne basse indique qu'il est plutôt en désaccord.

\section{Plan d'analyse des résultats}

Concernant les modes d'acculturation (assimilation, séparation, intégration et marginalisation), trois analyses ont été réalisées: (a) une analyse de fidélité ( $\alpha$ de Cronbach) afin de vérifier la stabilité des réponses des sujets à chacun des 12 items propres à chaque mode d'acculturation. Cette analyse de fidélité devrait 
permettre de déterminer si notre adaptation en français de l'échelle de Berry est un instrument fidèle pour mesurer les modes d'acculturation; (b) des analyses de variance (MANOVA) et de contraste ( $\mathrm{t}$ de Student) pour voir si on observait une variation de l'amplitude des attitudes des adolescents portugais en fonction des modes d'acculturation et, si oui, entre quels modes; (c) des analyses corrélationnelles ( $\mathrm{r}$ de Bravais-Pearson) pour examiner le degré de liaison entre les quatre modes d'acculturation. Enfin, concernant les relations entre les indicateurs scolaires et les quatre modes d'acculturation, des analyses de variance (MANOVA) ont été réalisées.

\section{Résultats}

La présentation et l'interprétation des résultats sont divisées en deux parties. Dans la première partie, nous discutons des résultats en rapport avec les quatre modes d'acculturation. Dans la deuxième partie, nous analysons la relation entre chacun des indicateurs scolaires (input, processus, output) et chacun des quatre modes d'acculturation (assimilation, séparation, intégration, marginalisation).

\section{Identité culturelle}

L'analyse de fidélité montre que les sujets ont tendance à donner des réponses légèrement plus homogènes aux items d'intégration, d'assimilation et de séparation $(\alpha$ moyen $=.75)$ qu'aux items de marginalisation $(\alpha=.62)$. Cette première analyse montre que notre adaptation de l'échelle de Berry est un instrument fidèle pour mesurer les modes d'acculturation des élèves migrants. Cette analyse de fidélité montre que les 12 affirmations (48 items en tout) propres à chacun des quatre modes d'acculturation mesurent sensiblement la même dimension.

Comme le montre le tableau 2, pour une majorité des sujets le mode d'acculturation le plus accepté est l'intégration (98\%) et celui le plus rejeté la séparation $(82 \%)$. L'assimilation est le mode le plus rejeté seulement par une minorité de sujets (16\%). La marginalisation est le mode le plus accepté ou le plus rejeté par une infime proportion de sujets (respectivement $2 \%$ et $2 \%$ ).

\section{Tableau 2: Pourcentage des sujets en fonction du mode d'acculturation le plus} accepté et le plus rejeté $(n=83)$

\begin{tabular}{|l|cccc|}
\hline Mode & Assimilation & Séparation & Intégration & Marginalisation \\
\hline Le plus accepté & 0 & 0 & 98 & 2 \\
Le plus rejeté & 16 & 82 & 0 & 2 \\
\hline
\end{tabular}

L'analyse de variance montre une variation significative $(F(3,246)=262.09$ p.<01) des attitudes en fonction du mode d'acculturation (tableau 3). Les sujets ont une attitude défavorable vis-à-vis de la séparation et, dans une moindre mesure, à l'égard de l'assimilation. Ils ont une attitude neutre envers la marginalisa- 
tion et clairement favorable vis-à-vis de l'intégration. Des analyses de contraste montrent que toutes ces attitudes sont significativement différentes les unes par rapport aux autres.

Tableau 3: Attitude moyenne et écart-type par mode d'acculturation (n=83)

\begin{tabular}{|cccc|}
\hline Assimilation & Séparation & Intégration & Marginalisation \\
\hline $2.82(.26)$ & $2.24(.62)$ & $3.97(.41)$ & $3.02(.48)$ \\
\hline
\end{tabular}

Une chose est de montrer qu'il existe des variations d'attitude significatives en fonction du mode d'acculturation, une autre est de voir si ces modes covarient. Les analyses corrélationnelles montrent que les adolescents portugais semblent traiter ces modes de façon relativement indépendante. En effet, mis à part l'assimilation et la marginalisation, et seulement pour la moitié des sujets (46\%), toutes les autres corrélations montrent que la majorité des sujets (entre $81 \%$ et $96 \%$ ) ont tendance à traiter séparément chacun de ces quatre modes (tableau 4). Ce sont les deux modes qui donnent lieu aux attitudes les moins marquées (neutre, marginalisation et assimilation) qui corrèlent le plus et de façon positive, tandis que ceux qui donnent lieu aux attitudes les plus extrêmes (pro-intégration et anti-séparation) sont en même temps ceux qui corrèlent le moins et de façon négative.

Tableau 4: Corrélations ( $r$ de Bravais-Pearson) entre les quatre modes d'acculturation $(n=83)$

\begin{tabular}{|l|ccc|}
\hline Mode & Séparation & Intégration & Marginalisation \\
\hline Assimilation & $.39^{* * *}$ & $.28^{* *}$ & $.68^{* * *}$ \\
Séparation & & $-.20^{*}$ & $.44^{* * *}$ \\
Intégration & & & $.20^{*}$ \\
\hline
\end{tabular}

Note: ${ }^{* * *}=\mathrm{p}<.01,{ }^{* *}=\mathrm{p}<.05$ et $^{*}=\mathrm{p}<.1$

\section{Identité culturelle et scolarité}

\section{Identité culturelle et indicateurs d'input}

Sexe: nous pouvons constater tout d'abord une surreprésentation dans notre échantillon des filles (60\%) par rapport aux garçons (40\%), ces derniers semblant montrer plus de réticences à répondre à des questions relativement personnelles sur leur identité culturelle. Les résultats (tableau 5) montrent que tant les garçons que les filles optent principalement pour l'intégration, contre la séparation et, dans une moindre mesure, contre l'assimilation, et qu' ils ont une attitude neutre vis-à-vis de la marginalisation. Nous ne relevons pas de différences significatives en fonction du sexe pour chacun des modes d'acculturation à l'exception de l'assimilation, les filles ayant une attitude significativement plus défavorable à l'égard de l'assimilation que les garçons $(\mathrm{F}(1,81)=3.52, \mathrm{p}<.10)$.

Age d'arrivée en Suisse: comme l'indique le tableau 5, la plupart des élèves $(83 \%)$ arrivent en Suisse en cours de scolarité, dont une majorité (68\%) en âge 
de fréquenter l'école obligatoire (primaire ou secondaire inférieure). Quel que soit leur âge d'arrivée, les élèves sont pour l'intégration, contre la séparation et, dans une moindre mesure, contre l'assimilation. Cependant, nous constatons des différences significatives dans l'amplitude des attitudes d'acculturation en fonction de la date d'arrivée en Suisse: les élèves arrivant en âge de fréquenter l'école secondaire inférieure (et qui sont donc arrivés le plus récemment) sont, d'une part, moins défavorables à l'assimilation $(F(3,73)=3.42, \mathrm{p}<.05)$ et, d'autre part, plus favorables à l'intégration $(\mathrm{F}(3,73)=2.56, \mathrm{p}<10)$ mais aussi à la marginalisation $(\mathrm{F}(3,73)=2.59, \mathrm{p}<.10)$ que les sujets arrivés avant cette date.

Tableau 5: Attitude moyenne et écart-type par mode d'acculturation en fonction d'indicateurs d'input (caractéristiques des élèves)

\begin{tabular}{|l|cccc|}
\hline \multirow{2}{*}{$\begin{array}{l}\text { Sexe } \\
\text { Garçons (n=33) }\end{array}$} & Assimilation & Séparation & Intégration & Marginalisation \\
\cline { 2 - 5 } Filles (n=50) & $2.88(.29)$ & $2.30(.55)$ & $3.95(.47)$ & $3.07(.52)$ \\
\hline $\begin{array}{l}\text { Age d'arrivée } \\
\text { Avant l'âge de fréquenter } \\
\text { l'école enfantine ( } \mathrm{n}=13)\end{array}$ & $2.77(.23)$ & $2.21(.67)$ & $3.99(.37)$ & $2.99(.45)$ \\
$\begin{array}{l}\text { En âge de fréquenter } \\
\text { l'école enfantine ( } \mathrm{n}=12)\end{array}$ & $2.74(.31)$ & $2.08(.73)$ & $3.94(.36)$ & $2.90(.41)$ \\
$\begin{array}{l}\text { En âge de fréquenter } \\
\text { l'école primaire }(\mathrm{n}=33)\end{array}$ & $2.70(.26)$ & $2.00(.43)$ & $3.74(.35)$ & $2.80(.57)$ \\
$\begin{array}{l}\text { En âge de fréquenter } \\
\text { l'école second. inf. }(\mathrm{n}=19)\end{array}$ & $2.80(.23)$ & $2.43(.67)$ & $3.95(.44)$ & $3.06(.48)$ \\
\hline
\end{tabular}

Niveau de formation du pèrelde la mère: une nette majorité des pères (88\%) et des mères $(95 \%)$ des élèves n'ont pas de formation professionnelle, la plupart ayant tout au plus achevé la scolarité obligatoire (respectivement 67\% et 69\%). Quel que soit le niveau de formation du père ou de la mère (tableau 6), les élèves sont clairement pour l'intégration, contre la séparation et ont tendance à s'opposer à l'assimilation et à avoir une attitude relativement neutre vis-à-vis de la marginalisation ${ }^{2}$. Enfin, il n'y a pas de variation significative de l'amplitude des attitudes des sujets vis-à-vis de chacun des quatre modes d'acculturation en fonction du niveau de formation de leur père ou de leur mère.

Permis de travail du pèrelde la mère: une très forte majorité d'élèves (92\%) ont un père détenteur actuellement d'un permis $C$ (tableau 6). En regard de l'échantillon examiné avec le premier questionnaire $(\mathrm{n}=294)$, nous pouvons relever une surreprésentation de permis $\mathrm{C}$. Aussi une analyse de la variation de l'amplitude des attitudes d'acculturation du sujet en fonction du permis actuel de leur père n'est-elle pas possible. Nous pouvons tout au plus observer des attitudes pro-intégration, anti-assimilation et surtout anti-séparation et neutres vis-à-vis de la marginalisation chez les sujets dont le père est détenteur d'un permis $C$.

Il en va différemment pour les mères des élèves. Bien que la plupart (79\%) soient actuellement au bénéfice d'un permis C, $18 \%$ ont un permis $B$. Les résul- 
tats montrent que, quel que soit le type de permis de la mère, les élèves optent principalement pour l'intégration, contre la séparation et, dans une moindre mesure, contre l'assimilation. Nous pouvons néanmoins relever des différences significatives en fonction du type de permis. Ainsi les élèves dont la mère a un permis $\mathrm{C}$ sont plus défavorables à l'égard de la marginalisation $(\mathrm{F}(3,68)=3.27$, $\mathrm{p}<.05)$ et de la séparation $(\mathrm{F}(3,68)=3.06, \mathrm{p}<.05)$ que les élèves dont la mère a un permis $\mathrm{B}$.

Tableau 6: Attitude moyenne et écart-type par mode d'acculturation en fonction d'indicateurs d'input (caractéristiques des familles d'élèves)

\begin{tabular}{|c|c|c|c|c|}
\hline Niv. de formation & Assimilation & Séparation & Intégration & Marginalisation \\
\hline $\begin{array}{l}\text { Aucune formation } \\
\text { scolaire achevée } \\
\text {-père }(n=11) \\
\text {-mère }(n=15)\end{array}$ & $\begin{array}{l}2.84(.29) \\
2.77(.27)\end{array}$ & $\begin{array}{l}2.17(.59) \\
1.93(.35)\end{array}$ & $\begin{array}{l}4.10(.40) \\
3.94(.47)\end{array}$ & $\begin{array}{l}3.12(.45) \\
3.04(.36)\end{array}$ \\
\hline $\begin{array}{l}\text { Scol. obl. achevée } \\
\text {-père }(n=50) \\
\text {-mère }(n=52)\end{array}$ & $\begin{array}{l}2.81(.24) \\
2.83(.24)\end{array}$ & $\begin{array}{l}2.34 \\
2.36(.68)\end{array}$ & $\begin{array}{l}3.95(.36) \\
3.98(.36)\end{array}$ & $\begin{array}{l}3.02(.48) \\
3.02(.49)\end{array}$ \\
\hline $\begin{array}{l}\text { Apprentissage } \\
\text {-père }(n=7) \\
- \text { mère }(n=2)\end{array}$ & $\begin{array}{l}2.88(.31) \\
3.14(.26)\end{array}$ & $\begin{array}{l}2.00(.47) \\
2.42(.35)\end{array}$ & $\begin{array}{l}3.98(.37) \\
3.67(.11)\end{array}$ & $\begin{array}{l}2.82(.51) \\
3.75(.35)\end{array}$ \\
\hline $\begin{array}{l}\text { Maturité } \\
\text {-père }(n=5) \\
\text {-mère }(n=3)\end{array}$ & $\begin{array}{l}2.81(.33) \\
2.95(.13)\end{array}$ & $\begin{array}{l}2.23(.55) \\
2.47(.63)\end{array}$ & $\begin{array}{l}4.05(.61) \\
3.86(.33)\end{array}$ & $\begin{array}{l}3.23(.56) \\
3.08(.46)\end{array}$ \\
\hline $\begin{array}{l}\text { Form prof. sup. } \\
\text {-père, }(n=2) \\
\text {-mère, }(n=2)\end{array}$ & $\begin{array}{l}2.93(.21) \\
3.04(.05)\end{array}$ & $\begin{array}{l}1.79(.05) \\
2.13(.53)\end{array}$ & $\begin{array}{l}3.96(.05) \\
4.42(.70)\end{array}$ & $\begin{array}{l}2.96(.17) \\
3.21(.53)\end{array}$ \\
\hline $\begin{array}{l}\text { Université } \\
\text {-père }(n=0) \\
- \text { mère }(n=1)\end{array}$ & $\begin{array}{l}- \\
2.45(-)\end{array}$ & $\begin{array}{l}- \\
1.33(-)\end{array}$ & $\begin{array}{l}- \\
4.17(-)\end{array}$ & $2.17(-)$ \\
\hline $\begin{array}{l}\text { Type de permis } \\
\text { Permis A } \\
\text { - père }(\mathrm{n}=0) \\
\text {-mère }(\mathrm{n}=1)\end{array}$ & $\begin{array}{l}- \\
2.45(-)\end{array}$ & $\begin{array}{l}- \\
1.33(-)\end{array}$ & $\begin{array}{l}- \\
4.17(-)\end{array}$ & $\begin{array}{l}- \\
2.16(-)\end{array}$ \\
\hline $\begin{array}{l}\text { Permis } B \\
\text {-père }(n=5) \\
\text {-mère }(n=13)\end{array}$ & $\begin{array}{l}2.88(.36) \\
2.87(.26)\end{array}$ & $\begin{array}{l}2.28(.90) \\
2.52(.57)\end{array}$ & $\begin{array}{l}3.95(.21) \\
3.96(.52)\end{array}$ & $\begin{array}{l}3.30(.65) \\
3.24(.48)\end{array}$ \\
\hline $\begin{array}{l}\text { Permis C } \\
\text {-père }(n=66) \\
\text {-mère }(n=58)\end{array}$ & $\begin{array}{l}2.80(.25) \\
2.80(.26)\end{array}$ & $\begin{array}{l}2.28(.62) \\
2.22(.62)\end{array}$ & $\begin{array}{l}3.96(.42) \\
3.96(.41)\end{array}$ & $\begin{array}{l}2.99(.46) \\
2.96(.43)\end{array}$ \\
\hline $\begin{array}{l}\text { Permis A } \\
\text { A eu un permis A } \\
\text {-père }(n=37) \\
\text {-mère }(n=15)\end{array}$ & $\begin{array}{l}2.81(.26) \\
2.81(.33)\end{array}$ & $\begin{array}{l}2.32(.69) \\
2.53(.81)\end{array}$ & $\begin{array}{l}3.98(.41) \\
3.86(.39)\end{array}$ & $\begin{array}{l}3.03(.43) \\
3.00(.49)\end{array}$ \\
\hline $\begin{array}{l}\text { N'a pas eu un permis } A \\
\text {-père }(n=37) \\
\text {-mère }(n=58)\end{array}$ & $\begin{array}{l}2.81(.26) \\
2.81(.25)\end{array}$ & $\begin{array}{l}2.20(.57) \\
2.19(.57)\end{array}$ & $\begin{array}{l}3.93(.43) \\
3.99(.41)\end{array}$ & $\begin{array}{l}2.92(.48) \\
2.99(.46)\end{array}$ \\
\hline
\end{tabular}

Comme on peut le voir au tableau $6,50 \%$ des élèves ont un père et $21 \%$ ont une mère qui ont d'abord bénéficié d'un permis $A$. Que le père ou la mère ait ou non bénéficié d'un permis $A$, nous voyons que les élèves optent principalement pour l'intégration, contre l'assimilation, mais surtout contre la séparation et ont une 
attitude neutre vis-à-vis de la marginalisation. Cependant, nous observons que les sujets dont la mère a eu un permis $\mathrm{A}$ sont moins opposés à l'égard de la séparation $(\mathrm{F}(1,71)=3.63, \mathrm{p}<.10)$.

En résumé, l'identité culturelle varie en fonction de trois des quatre indicateurs d'input retenus: le sexe a une incidence sur l'assimilation; l'âge d'arrivée en Suisse sur l'assimilation, l'intégration et la marginalisation; le permis de travail de la mère sur la séparation et la marginalisation. Par contre, le niveau de formation des parents n'a pas d'incidence sur l'identité culturelle et ce quel que soit le mode d'acculturation considéré.

\section{Identité culturelle et indicateurs de processus}

Redoublement: comme l'indique le tableau 7, la moitié des sujets ont redoublé au cours de leur scolarité obligatoire. Que les sujets aient redoublé ou pas, les résultats montrent qu'ils optent principalement pour l'intégration, contre la séparation et, dans une moindre mesure, contre l'assimilation et qu'ils ont une attitude neutre vis-à-vis de la marginalisation. Cependant, nous observons une variation significative de l'amplitude des attitudes de séparation $(\mathrm{F}(1,78)=4.35$ $\mathrm{p}<.05)$ et d'intégration $(\mathrm{F}(1,78)=3.66 \mathrm{p}<.10)$. Les sujets qui ont redoublé ont tendance à être moins en défaveur de la séparation et moins en faveur de l'intégration que les sujets qui n'ont jamais redoublé.

Changement de division: seuls $16 \%$ des sujets ont changé positivement de division au cours de leur scolarité (tableau 7). Que les sujets aient ou non changé de division, les résultats montrent qu'ils sont pour l'intégration, contre la séparation et, dans une moindre mesure, contre l'assimilation et qu'ils ont une attitude neutre vis-à-vis de la marginalisation. Néanmoins, mis à part l'intégration $(\mathrm{F}(1,78)=6.17 \mathrm{p}<.05)$, il n'y a pas de variation significative de l'amplitude des attitudes d'acculturation en fonction du changement de division: on observe que les sujets qui n'ont jamais eu l'occasion de changer de niveau d'enclassement sont moins en faveur de l'intégration que leurs camarades qui ont changé de niveau.

Fréquentation des cours de langue et de civilisation portugaises: la moitié des élèves ont suivi les cours de langue et de civilisation portugaises (tableau 7). Qu'ils aient suivi ou non ces cours, les élèves optent principalement pour l'intégration et contre la séparation et, dans une moindre mesure, contre l'assimilation. De plus, ils sont neutres vis-à-vis de la marginalisation. Cependant les élèves ayant suivi ces cours sont plus défavorables à l'égard de l'assimilation $(\mathrm{F}(1,76)=3.02, \mathrm{p}<.10)$ et de la marginalisation $(\mathrm{F}(1,76)=2.74, \mathrm{p}<.10)$. 
Tableau 7: Attitude moyenne et écart-type par mode d'acculturation en fonction d'indicateurs de processus

\begin{tabular}{|l|cccc|}
\hline & Assimilation & Séparation & Intégration & Marginalisation \\
\cline { 2 - 5 } Redoublement & & & & \\
N'a pas redoublé $(\mathrm{n}=41)$ & $2.83(.25)$ & $2.09(.57$ & $4.05(.38)$ & $3.03(.50)$ \\
A redoublé $(\mathrm{n}=42)$ & $2.81(.27)$ & $2.39(.63)$ & $3.89(.42)$ & $3.00(.45)$ \\
\hline Changement de div & & & \\
N'a pas changé $(\mathrm{n}=70)$ & $2.83(.25)$ & $2.26(.63)$ & $3.92(.42)$ & $3.03(.47)$ \\
A changé $(\mathrm{n}=13)$ & $2.78(.27)$ & $2.17(.59)$ & $4.20(.23)$ & $2.95(.49)$ \\
\hline Fréquentation des & & & \\
cours de langue... & & & \\
N'a pas suivi $(\mathrm{n}=38)$ & $2.88(.24)$ & $2.23(.61)$ & $3.95(.46)$ & $3.10(.47)$ \\
A suivi $(\mathrm{n}=38)$ & $2.77(.27)$ & $2.19(.62)$ & $4.00(.38)$ & $2.93(.47)$ \\
\hline
\end{tabular}

En résumé, l'identité culturelle varie selon les trois indicateurs de processus retenus: le redoublement a une incidence sur la séparation et l'intégration; le changement de division sur l'intégration; la fréquentation des cours de langue et civilisation portugaises sur l'assimilation et la marginalisation.

\section{Identité culturelle et indicateurs d'output}

Niveau d'enclassement final: une majorité des élèves (60\%) se trouvent dans le niveau d'enclassement le plus bas à la fin de leur scolarité et une minorité dans le niveau intermédiaire (30\%) ou le plus élevé (10\%) (tableau 8). Quel que soit le niveau d'enclassement final, les élèves ont des attitudes pro-intégration et anti-séparation et, dans une moindre mesure, anti-assimilation et neutre vis-à-vis de la marginalisation. Pour tous les modes d'acculturation, il n'y pas de variation significative de l'amplitude des attitudes en fonction du niveau d'enclassement final.

Projet de formation: une forte majorité des élèves $(86 \%)$ ont un projet de formation. Quel que soit le mode d'acculturation que l'on considère, il n'y a pas de variation significative de l'amplitude des attitudes en fonction de la présence ou de l'absence d'un projet de formation (tableau 8); tous les sujets optent principalement pour l'intégration, contre la séparation et, dans une moindre mesure, contre l'assimilation et sont neutres vis-à-vis de la marginalisation.

\section{Tableau 8: Attitude moyenne et écart-type par mode d'acculturation en fonction} d'indicateurs d'output

\begin{tabular}{|l|cccc|}
\hline \multirow{2}{*}{ Enclassement final } & Assimilation & Séparation & Intégration & Marginalisation \\
\cline { 2 - 5 } I $(\mathrm{n}=50)$ & $2.83(.24)$ & $2.34(.63)$ & $3.97(.46)$ & $3.06(.44)$ \\
II $(\mathrm{n}=25)$ & $2.78(.29)$ & $2.09(.54)$ & $3.99(.35)$ & $2.97(.55)$ \\
III $(\mathrm{n}=8)$ & $2.85(.28)$ & $2.15(.72)$ & $3.94(.19)$ & $2.90(.45)$ \\
\hline Projet de formation & & & & \\
A un projet $(\mathrm{n}=70)$ & $2.28(.26)$ & $2.23(.62)$ & $3.97(.42)$ & $3.00(.52)$ \\
N'a pas de projet $(\mathrm{n}=11)$ & $2.77(.24)$ & $2.39(.69)$ & $3.98(.39)$ & $3.14(.30)$ \\
\hline
\end{tabular}

En résumé, l'identité culturelle ne varie pas en fonction des deux indicateurs d'output retenus et ce quel que soit le mode d'acculturation considéré. 


\section{Identité culturelle et niveau général de réussite scolaire}

Mis à part le type de cursus scolaire "Moyen», on observe une relation inversement proportionnelle entre le niveau de réussite du cursus scolaire (du type «Très faible» au type "Très bon») et la proportion d'adolescents portugais se trouvant dans chacun de ces niveaux: $31 \%$ des sujets ont un cursus scolaire "Très faible», 29\% un cursus "Faible», 8\% un cursus "Moyen», 22\% un cursus "Bon» et seulement $10 \%$ un cursus "Très bon».

Quel que soit le niveau général de réussite scolaire (tableau 9), les sujets sont légèrement opposés à l'assimilation, clairement contre la séparation, neutres visà-vis de la marginalisation et franchement en faveur de l'intégration. Il existe cependant une variation significative de l'amplitude des attitudes de séparation $(\mathrm{F}(4,78)=2.31 \mathrm{p}<.10)$ et d'intégration $(\mathrm{F}(4,78)=2.02 \mathrm{p}<.10)$ en fonction du niveau général de réussite scolaire. Les sujets dont le niveau est le plus bas (type "Très faible») sont ceux qui sont le moins en défaveur de la séparation et le moins en faveur de l'intégration, les autres niveaux donnant lieu à des attitudes de séparation et d'intégration significativement non différentes.

Tableau 9: Attitude moyenne et écart-type par mode d'acculturation en fonction du niveau général de réussite scolaire

\begin{tabular}{|l|cccc|}
\hline Niveau de réussite & Assimilation & Séparation & Intégration & Marginalisation \\
\hline Très faible $(\mathrm{n}=26)$ & $2.79(.26)$ & $2.54(.68)$ & $3.81(.45)$ & $2.99(.47)$ \\
Faible $(\mathrm{n}=24)$ & $2.88(.30)$ & $2.11(.29)$ & $4.12(.33)$ & $3.15(.42)$ \\
Moyen $(\mathrm{n}=7)$ & $2.89(.30)$ & $2.02(.29)$ & $3.91(.33)$ & $3.14(.42)$ \\
Bon $(\mathrm{n}=18)$ & $2.74(.29)$ & $2.11(.62)$ & $4.02(.36)$ & $2.91(.59)$ \\
Très bon $(\mathrm{n}=8)$ & $2.85(.28)$ & $2.15(.32)$ & $3.94(.19)$ & $2.90(.45)$ \\
\hline
\end{tabular}

En résumé, l'identité culturelle varie en fonction du niveau général de réussite scolaire. Ce niveau a une incidence sur la séparation et l'intégration.

\section{Discussion}

\section{Scolarité de l'élève migrant portugais}

Rappelons que l'objectif de ce travail est d'analyser la relation éventuelle entre certains indicateurs scolaires relatifs au sujet, à sa famille et à son cursus scolaire d'une part, et relatifs à l'identité culturelle du sujet d'autre part.

Il convient tout d'abord de relever que l'intégration scolaire des élèves portugais est particulièrement problématique. Ainsi, une majorité d'élèves se trouvent, à la fin de leur scolarité obligatoire, dans le niveau d'enclassement le plus bas (parmi ces élèves une partie se trouvent dans des classes regroupant des élèves en difficulté). Leurs possibilités de formation post-obligatoire et donc de débouchés professionnels sont des plus limités. Une moitié des élèves ont redoublé au moins une fois durant leur scolarité, ce qui est un indice de difficultés scolaires impor- 
tantes. Seule une petite minorité d'élèves ont eu l'occasion de changer de division positivement au cours de l'école obligatoire, autrement dit de valoriser leurs capacités intellectuelles.

Ce constat d'échec est renforcé quand nous comparons le cursus scolaire de l'adolescent portugais à celui de l'ensemble des adolescents, toutes nationalités confondues, en fin de scolarité obligatoire (Doudin, 1996a). En effet, sur l'ensemble de la population, d'une part une proportion nettement plus faible d'élèves se trouvent dans le niveau d'enclassement final le plus bas ou redoublent de degré au cours de leur scolarité et, d'autre part, une proportion nettement plus importante changent positivement de division au cours du secondaire inférieur.

Sur la base de ces résultats, nous pouvons donc affirmer qu'une majorité des adolescents portugais sont fortement marginalisés sur le plan scolaire. Cette marginalisation peut avoir des conséquences particulièrement négatives: elle peut déboucher sur une marginalisation professionnelle (par ex. difficulté d'accéder à une formation professionnelle ou alors dans des métiers peu valorisés), voire sociale (par ex. bas salaire, risque accru de chômage). Mais, est-ce que cette marginalisation scolaire des adolescents portugais entretient un lien avec leur identité culturelle?

\section{Identité culturelle de l'élève migrant portugais}

Il convient tout d'abord de relever que, sur l'ensemble de l'échantillon étudié, les résultats montrent très clairement que les adolescents portugais en fin de scolarité obligatoire ont tendance, d'une part, à vouloir préserver leur culture d'origine tout en intégrant en même temps la culture suisse (attitude clairement prointégration), et, d'autre part, sont contre un rejet de la culture suisse (attitude clairement anti-séparation) et portugaise (attitude légèrement anti-assimilation). Ce résultat concorde avec celui obtenu aux Pays-Bas par van de Vijver, HelmsLorez et Feltzer (1999) chez des élèves migrants de différentes origines. Leur attitude neutre à l'égard de la marginalisation peut s'expliquer par le fait que cette dimension ne fait pas sens à leurs yeux. En effet, il faut rappeler que la marginalisation a été repérée par Berry, Kalin et Taylor (1977), entre autres chez les Amérindiens du Canada, dont une des caractéristiques culturelles était la perte de tout cadre de référence (rejet non seulement des valeurs minoritaires traditionnelles ancestrales mais aussi des valeurs dominantes anglo-saxonnes contemporaines).

Étant donné leurs attitudes pro-intégration, anti-séparation et, dans une moindre mesure, anti-assimilation, nous pouvons en déduire que les adolescents portugais cherchent très clairement à s'intégrer à la culture de leur pays d'accueil tout en préservant leur identité culturelle d'origine, plutôt que de rejeter leur culture d'origine etlou celle de leur pays d'accueil. Ces résultats vont dans le même sens que ceux trouvés en France (Neto, 1994, 1995), au Canada ou en Australie (Berry et al., 1989; Lanca et al., 1994). 
Nous pouvons cependant nous demander si ces attitudes à l'égard des modes d'acculturation sont les mêmes relativement à chacun des indicateurs scolaires retenus et ce d'autant plus que ces différents modes semblent être traités par les adolescents portugais de façon relativement indépendante. En effet, les analyses corrélationnelles montrent qu'une attitude anti-séparation, par exemple, n’implique pas nécessairement une attitude pro-intégration et inversement.

\section{Identité culturelle et scolarité de L'élève migrant por- tugais}

Les résultats montrent clairement que, en fonction des caractéristiques du sujet (sexe, âge d'arrivée), de sa famille (niveau de formation du père/de la mère, permis de travail du père/de la mère) et de son cursus scolaire (redoublement, changement de division, fréquentation des cours de langue et de civilisation portugaises, enclassement final, projet de formation), il existe bien des différences entre sujets relativement à leur mode d'acculturation: même si les sujets optent pour une attitude pro-intégration, celle-ci peut néanmoins varier dans son intensité tout comme leurs attitudes anti-séparation, anti-assimilation et leur neutralité par rapport à la marginalisation. Ainsi, nous pouvons établir les profils-types suivants:

- le sujet optant le plus clairement pour un compromis entre les cultures suisse et portugaise (pro-intégration) est arrivé le plus récemment (en âge de fréquenter l'école secondaire inférieure), n'a jamais redoublé, a changé positivement de division au cours de sa scolarité, a un niveau général de réussite scolaire de moyen à très bon. Au contraire, le sujet qui opte pour ce compromis, mais de la manière la plus nuancée, est arrivé plus anciennement (avant l'âge de fréquenter l'école secondaire inférieure), a redoublé, n’a jamais changé de division, a un niveau général de réussite scolaire très faible;

- le sujet le plus opposé au rejet de la culture suisse en faveur de la culture portugaise (anti-séparation) n’a jamais redoublé, son niveau général de réussite scolaire va de moyen à très bon, sa mère n'a jamais eu de permis $\mathrm{A}$, elle est actuellement détentrice d'un permis $\mathrm{C}$. Au contraire, le sujet le moins opposé au rejet de la culture suisse en faveur de la culture portugaise a redoublé, son niveau général de réussite scolaire est très faible, sa mère a d'abord été détentrice d'un permis A, actuellement elle est détentrice d'un permis B;

- le sujet qui est le plus opposé à l'abandon de son identité portugaise en faveur de l'identité suisse (anti-assimilation) est une fille arrivée avant l'âge de fréquenter l'école secondaire, elle a suivi les cours de langue et de civilisation portugaises. Au contraire, le sujet qui est le moins opposé à l'abandon de son identité portugaise en faveur de l'identité suisse est un garçon arrivé récemment (en âge de fréquenter l'école secondaire inférieure), qui n’a pas suivi les cours de langue et de civilisation portugaises;

- le sujet qui est le plus opposé au rejet de toute culture suisse ou portugaise (anti-marginalisation) est arrivé avant l'âge de fréquenter le secondaire infé- 
rieur, il a suivi les cours de langue et de civilisation portugaises, sa mère est détentrice actuellement d'un permis C. Au contraire, le sujet qui est le moins opposé au rejet de ces deux cultures est arrivé récemment (en âge de fréquenter l'école secondaire inférieure), il n'a pas suivi de cours de langue et de civilisation portugaises, sa mère est détentrice actuellement d'un permis B.

Sur la base de ces résultats, nous pouvons avancer quelques interprétations. Tout d'abord, nous constatons que les deux caractéristiques du sujet retenues dans cette étude (le sexe et l'âge d'arrivée en Suisse) ont une incidence sur l'amplitude de ses attitudes vis-à-vis de l'intégration, de l'assimilation et/ou de la marginalisation.

Ainsi, les filles semblent plus opposées que les garçons à un rejet de leur culture portugaise en faveur de la culture suisse. Elles auraient une attitude plus loyale vis-à-vis de leur culture d'origine que les garçons. Il est possible que les filles portugaises reçoivent une éducation plus traditionnelle que les garçons et que, par conséquent, elles soient plus opposées à un rejet de la culture d'origine de leur famille.

Les élèves arrivés le plus récemment en Suisse, au contraire de ceux qui y séjournent depuis plus longtemps, ont une attitude paradoxale: tout en étant moins défavorables à un rejet de la culture portugaise en faveur de la culture suisse, et plus favorables à une valorisation des cultures portugaise et suisse, ils optent également (et même si c'est dans une moindre mesure) pour un rejet de ces deux cultures. Ce paradoxe peut avoir pour origine un conflit de loyauté. Loyauté vis-à-vis de leur passé d'une part: c'est au Portugal que les élèves arrivés le plus récemment en Suisse ont vécu la plus grande partie de leur vie, ils y ont construit leurs premiers liens sociaux et familiaux ainsi que leur première identité culturelle; loyauté vis-à-vis du choix de leurs parents de s'établir en Suisse, d'autre part, auquel vient peut-être s'ajouter une volonté d'adaptation rapide à un environnement différent (désir de conformité avec le groupe dominant). Si la première «solution» peut paraitre relativement adaptée, par contre la deuxième est plus problématique: elle peut être l'expression d'une fragilité de la construction de l'identité culturelle à laquelle il faut être très attentif.

Ensuite, concernant les caractéristiques familiales des adolescents portugais, nous pouvons relever l'absence d'influence du niveau de formation des parents sur leurs modes d'acculturation. Bien que cette variable joue un rôle fondamental, notamment dans la qualité de l'intégration scolaire de l'élève (voir Doudin, Pons et Moreau, 1998), elle ne semble pas influencer la qualité de son identité culturelle.

Par contre le permis de travail de la mère joue une rôle important. Si le lien entre le type de permis $\mathrm{B}$ et $\mathrm{C}$ du père et les modes d'acculturation de l'enfant n'a pas pu être analysé du fait de la composition de notre échantillon (voir tableau 6), les résultats montrent l'absence de lien significatif entre le fait que le père ait été détenteur ou non d'un permis A et l'identité culturelle de l'enfant. Par contre, les adolescents dont la mère a eu un permis $\mathrm{A}$, ainsi que les adolescents dont la 
mère est actuellement détentrice d'un permis B sont moins défavorables à un rejet de la culture suisse en faveur de la culture portugaise que ceux dont la mère n'a jamais eu de permis A ou qui est actuellement en possession d'un permis C. De plus, ils sont légèrement favorables à un rejet de ces deux cultures (promarginalisation), ce qui n'est pas le cas des adolescents dont la mère a actuellement un permis C. Ces derniers sont très légèrement défavorables au rejet de ces deux cultures. Le fait que la mère ait été détentrice d'un permis A implique que l'enfant a, soit été séparé de sa mère durant une plus ou moins longue période (rupture partielle des liens mère-enfant), soit vécu clandestinement en Suisse. Ces deux situations engendrées par ce type de permis ont donc des conséquences néfastes, même si relatives, au niveau de l'identité culturelle de l'enfant, celui-ci ayant plus tendance à se replier sur sa propre culture ou à se couper de toute culture. Ces résultats montrent également le rôle fondamental de la relation mère-enfant, si souvent souligné en psychologie.

Enfin, lorsque l'on examine les relations entre les modes d'acculturation et les différents indicateurs de cursus scolaire retenus, nous constatons que, mis à part le niveau d'enclassement final et le projet de formation post-obligatoire, les autres indicateurs (redoublement, changement de division, niveau général de réussite scolaire et fréquentation des cours de langue et civilisation portugaises) entretiennent une relation avec les attitudes des adolescents portugais vis-à-vis de l'intégration, de la séparation, de l'assimilation ou de la marginalisation. Les adolescents en difficulté scolaire, autrement dit qui ont redoublé, qui n'ont jamais changé positivement de division et/ou dont le niveau général de réussite scolaire est globalement faible, sont moins en faveur d'une valorisation des cultures suisse et portugaise (attitudes pro-intégration moins marquées) et sont moins opposés à un rejet de la culture suisse en faveur de la culture portugaise (attitudes anti-séparation moins marquées). Sans préjuger de la relation d'implication entre l'échec scolaire et l'identité culturelle (voir ci-après), nous constatons que la marginalisation scolaire (avec les conséquences possibles d'une marginalisation professionnelle, voire sociale) s'accompagne, même si de façon relative, d'un repli sur les valeurs de la culture d'origine, voire d'un certain flou de l'identité culturelle. Même si nous trouvons des fléchissements et non pas des renversements de tendance, nos résultats vont bien dans le même sens que ceux de Bastide (1994).

En ce qui concerne plus précisément les cours de langue et civilisation portugaises, nous constatons que les sujets qui ont fréquenté ces cours, au contraire de ceux qui ne les ont pas fréquentés, sont plus défavorables à un rejet de la culture portugaise (attitude anti-assimilation plus marquée) et qu'ils s'opposent, même si c'est de façon légère, à un rejet des cultures suisse et portugaise (attitude antimarginalisation). Ce type de cours semble donc jouer un rôle particulièrement favorable auprès des élèves qui les fréquentent puisque ceux-ci, en enrichissant le lien avec leur culture d'origine, ont tendance a éviter la marginalisation (voir leur attitude vis-à-vis du rejet des cultures suisse et portugaise). 


\section{Conclusion}

Rappelons tout d'abord que nous n'observons pas de renversement, mais tout au plus des fléchissements des attitudes pro-intégration (valorisation des cultures suisse et portugaise), anti-assimilation (contre le rejet de la culture portugaise en faveur de la culture suisse) et anti-séparation (contre le rejet de la culture suisse en faveur de la culture portugaise), et neutre relativement à la marginalisation (neutralité vis-à-vis du rejet des cultures suisse et portugaise) et ceci quel que soit l'indicateur scolaire. Ces fléchissements méritent néanmoins une attention particulière. Du fait qu'ils ne sont que des fléchissements, ils démontrent l'existence chez l'adolescent portugais d'une identité culturelle adaptée et relativement solide. Malgré leurs difficultés d'intégration scolaire, les adolescents portugais sont en faveur d'une valorisation des cultures suisse et portugaise et opposés au rejet d'une de ces deux cultures en faveur de l'autre. Ces fléchissements peuvent être toutefois les signes avant-coureurs de problèmes d'adaptation auxquels il faut être attentif.

Bien qu'il soit difficile, voire impossible, d'intervenir au niveau des caractéristiques personnelles et familiales de l'adolescent portugais, une intervention au niveau du contexte scolaire paraît, par contre, indispensable. Mais une intervention au niveau scolaire soulève le problème du sens de la relation implicative entre modes d'acculturation et indicateurs scolaires. Si, pour les caractéristiques de l'élève et de sa famille, il est relativement facile de définir le sens de cette relation, par contre, concernant les indicateurs scolaires de processus et d'output, il est plus difficile de dire qui, des indicateurs ou des modes d'acculturation, est la cause ou la conséquence de l'autre. En fonction de notre plan expérimental, il n'est pas possible de répondre à cette question. Nous pouvons toutefois nous demander si l'implication se situe à un niveau aussi linéaire ou si ces phénomènes ne se surdétermineraient pas (causalité circulaire). Quoi qu'il en soit, il nous semble que la marginalisation scolaire, cause et/ou conséquence, se retrouve sur le plan des modes d'acculturation. Le risque est alors grand qu'un cercle vicieux s'installe avec des conséquences négatives pour le développement de l'adolescent.

Il convient donc que l'école mette tout en œuvre pour casser ce cercle vicieux, d'une part en favorisant toute intervention qui permettrait la construction d'une identité culturelle adaptée, par exemple la fréquentation des cours de langue et de civilisation portugaises et, d'autre part, en évitant de recourir à des mesures d'aide de nature paradoxale. Comme nous l'avons déjà relevé au début de ce travail, les mesures d'aide traditionnellement prises par l'école (redoublement, appui pédagogique hors classe, regroupement des élèves en difficulté dans un même type de classe) reposent toutes sur le paradoxe qui consiste à vouloir mieux intégrer scolairement un élève tout en l'excluant de son groupe d'âge et/ou de la classe régulière. Afin de dépasser ce paradoxe, de nouvelles approches sont tentées (par ex. Acheson et Gall, 1992/1993; Saint-Laurent et al., 1995; Doudin, 1996b) comme la supervision pédagogique. En fournissant aux enseignants no- 
tamment un feed-back sur leur manière d'enseigner, la supervision leur permet de développer des stratégies d'enseignement plus efficaces pour l'ensemble de la classe, pour un sous-groupe d'élèves ou pour un élève en particulier, et d'éviter ainsi le recours à l'exclusion.

Enfin, les effets nocifs du permis A (rupture de certains liens familiaux et plus particulièrement de la relation mère-enfant) et $B$ (précarité du statut) se confirment (voir également Cattafi-Maurer et al., 1999; Doudin, Pons et Moreau, 1998). Le problème est ici de dimension politique et il convient de dénoncer avec force le statut juridique lié à ces types de permis et, plus généralement, toute politique coercitive du groupe majoritaire vis-à-vis des groupes minoritaires.

\section{Notes}

1 Rappelons qu'en Suisse, il existe trois types de permis principaux pour les travailleurs migrants: A, B et C. Le permis A offre le statut juridique le plus précaire. Le permis $\mathrm{C}$ offre le plus d'avantages et permet, sous certaines conditions, le regroupement familial.

2 Seuls les deux premiers niveaux de formation («aucune formation achevée» et «scolarité obligatoire achevée») peuvent être analysés statistiquement étant donné que, pour les autres niveaux, les effectifs sont insuffisants.

\section{Bibliographie}

Acheson, K.A., \& Gall, M.D. (1992). Techniques in the clinical supervision of teachers. New York: Longman. Trad. fr. (1993). La supervision pédagogique: méthodes et secrets d'un superviseur clinicien. Montréal: Editions Logiques.

Arroteia, J., \& Doudin, P.-A. (Eds.). (1998). Trajectórias sociais e culturais de jovens portugueses no espaço europeu: questōes multiculturais e de integração. Aveiro: Universidade de Aveiro.

Baldauf, R.B., \& Ayabe, H.I. (1977). Acculturation and educational achievement in American Samoan adolescents. Journal of Cross-Cultural Psychology, 8, 2, 241-256.

Bastide, L. (1994). Identité culturelle et intégration sociale et scolaire de l'échantillon étudié. In D. Becker, M.-E. Handman \& R. Ittura (Eds.), Échec scolaire ou école en échec (pp. 133171). Paris: L'Harmattan.

Berry, J.W. (1989). Acculturation et adaptation psychologique. In J. Retschitzky, M. BosselLargos \& P. Dasen (Eds.), La recherche interculturelle. Tome 1 (pp. 135-145). Paris: L'Harmattan.

Berry, J.W. (1995). Psychology of aculturation. In N.R. Goldberg, J.B. Veroff (Eds.), The culture and psychology reader (pp. 457-488). New York: New York University Press.

Berry, J.W. (1999). Intercultural relations in plural societies. Canadian Psychology, 40, 1, $12-$ 21.

Berry, J., \& Sam, D. (1997). Acculturation and adaptation. In J. Berry, M. Segall \& C. Kagitcibasi (Eds.), Handbook of cross-cultural psychology, Vol. 3 (pp. 291-236). Boston: Allyn \& Bacon.

Berry, J.W., Kalin, R., \& Taylor, D.M. (1977). Multiculturalism and ethnic attitudes in Canada. Ottawa: Government of Canada.

Berry, J.W, Klim, U., Power, S., \& Young, M. (1989). Acculturations attitudes in plural societies. Applied Psychology: An International Review, 38, 2, 185-206. 
Bottani, N., \& Walberg, H.J. (1992). A quoi servent les indicateurs internationaux de l'enseignement? In L'OCDE et les indicateurs internationaux de l'enseignement (pp. 7-13). Paris: CERI.

Deschamps, J.-C. (1991). Définition de soi et identité sociale. In W. Dois, J.-C. Deschamps \& G. Mugny, Psychologie sociale expérimentale (pp. 35-51). Paris: Armand Colin.

Cattafi-Maurer, F., Abriel, G., Dasen, P.R., Lack, C., \& Perregaux, C (1998). Viver em precariedade: o acesso a uma formação profissional dos jovens potugueses com estatuto precário. In J. Arroteia \& P.-A. Doudin (Eds.), Trajectorias sociais e culturais de jovens portugueses no espaço europeu: questôes multiculturais e de integração (pp. 183-203). Aveiro: Universidade de Aveiro.

Dona, G., \& Berry, J. (1994). Acculturation attitudes and acculturative stress of Central American refugees. International Journal of Psychology, 29, 1, 57-70.

Doudin, P.-A. (1996a). L'école vaudoise face aux élèves étrangers. Lausanne: CVRP.

Doudin, P.-A. (1996b). Élèves en difficultés: la pédagogie compensatoire est-elle efficace? Psychoscope, 17, 9, 4-7.

Doudin, P.-A. (1998). Scolarisation des enfants portugais en Suisse/Schulung der portugiesischen Kinder in der Schweiz. Berne: CDIP/EDK.

Doudin, P.-A., Pons, F., \& Moreau, J. (1998). Dificuldades de integração escolar dos alunos portugueses na Suiça: causas e remediaçōes. In J. Arroteia \& P.-A. Doudin (Eds.), Trajectorias sociais e culturais de jovens portugueses no espaço europeu: questoes multiculturais e de integração (pp. 157-182). Aveiro: Universidade de Aveiro.

Inkeles, A., \& Smith, D. (1974). Becoming modern: Individual change in six developing countries. Cambridge: Harvard University Press.

Krishnan, A., \& Berry, J. (1992). Acculturative stress and acculturation attitudes among Indian immigrants to the United States. Psychology and Developing Societies, 4, 2, 187-212.

Kuhn, M., \& McPartland, T. (1954). An empirical investigation of self-attitudes. American Sociological Review, 19, 68-76.

Lanca, M., Alksnis, C., Roese, N., \& Gardner, R. (1994). Effects of language choice on acculturation: A study of Portuguese immigrants in a multicultural setting. Journal of Language and Social Psychology, 13, 3, 315-330.

Neto, F. (1993). Quelques contributions de la recherche psychologique à la compréhension du phénomène migratoire portugais. In M. Rey von Allmen (Ed.), Psychologie clinique et interrogations culturelles (pp. 223-242). Paris: L'Harmattan.

Neto, F. (1994). Le stress d'acculturation chez des jeunes d'origine portugaise en France. Enfance, $1,83-94$.

Neto, F. (1995). Predictors of satisfaction with life among second generation migrants. Social Indicators Research, 35, 1, 93-116.

Nuttall, D. (1992). Les indicateurs internationaux de l'enseignement: leurs fonctions et leurs limites. In L'OCDE et les indicateurs internationaux de l'enseignement (pp. 15-25). Paris: CERI.

OCDE. (1996). Regards sur l'éducation. Les indicateurs de l'OCDE. Paris: CERI.

OFS (1995). Les indicateurs de l'enseignement en Suisse. L'enseignement en mutation dans notre pays. Berne: OFS.

Pérez, J.-A., Mugny, G., Llavata, E., \& Fierres, R. (1993). Paradoxe de la discrimination et conflit culturel: études sur le racisme. In J.-A. Pérez \& G. Mugny (Eds.), Influences sociales. La théorie de l'élaboration du conflit (pp.145-168). Neuchâtel: Delachaux \& Niestlé.

Saint-Laurent, L., Giasson, J., Simard, C., \& Dione, J. (1995). Programme d'intervention auprès des élèves à risques; une nouvelle option éducative. Montréal: Gaëtan Morin.

Silva, M.C., \& Neto, F. (1993). Psychosocial predictors of intent to emigrate among portuguese adolescents. Journal of Applied Social Psychology, 23, 16, 1285-1302.

Tajfel, H., Billing, M., Bundy, R., \& Flament, C. (1971). Social categorisation and intergroup behaviour. European Journal of Social Psychology, 1, 149-178. 
Turner, J. (1987). Rediscovering the social group. A self-categorisation theory. Oxford: Blackwell. van de Vijver, F., Helms-Lorenz, M., \& Feltzer, M. (1999). Acculturation and cognitive performance of migrant children in the Nederlands. International Journal of Psychology, 34, 3, 149-162.

Zavalloni, M., \& Louis-Guerin, C. (1984). Identité sociale et conscience: Introduction à l'égoécologie. Montréal: Presses de l'Université de Montréal.

\section{Kulturelle Identität und Beschulung portugiesischer Schüler in der Schweiz}

\section{Zusammenfassung}

In diesem Artikel untersuchen wir die Beziehung zwischen der kulturellen Identität und bestimmten persönlichen, familiären und schulischen Merkmalen von Migrantenkindern portugiesischer Herkunft, die ihre obligatorische Schulzeit in der Schweiz (Kanton Waadt) beenden. Die kulturelle Identität wird mit einer adaptierten Version der Akkulturationsskala von Berry gemessen. Die Ergebnisse zeigen, dass diese Schüler einerseits deutlich die Tendenz haben, ihre Ursprungskultur zu bewahren und gleichzeitig die Schweizer Kultur anzunehmen (klar pro-integrative Einstellung); andererseits sind sie sowohl gegen eine Ablehnung der Schweizer (deutlich antiseparatistische Einstellung) als auch der portugiesischen Kultur (leicht anti-assimilatorische Einstellung). Allerdings konnten wir in Abhängigkeit von bestimmten persönlichen und familiären Merkmalen (Geschlecht, Einreisedatum in die Schweiz bzw. Wiederholung eines Schuljahres, schulisches Leistungsniveau, Besuch von portugiesischen Sprach- und landeskundlichen Kursen) signifikante Abschwächungen bestimmter dieser Haltungen feststellen, besonders der pro-integrativen Einstellung. Diese Ergebnisse werden in bezug auf ihre Konsequenzen auf schulischer und poliitischer Ebene (Gefahr von schulischer bzw. sozialer Marginalisierung) diskutiert.

\section{Identità culturale e scolarizzazione di allievi portoghesi in Svizzera}

\section{Riassunto}

In questo articolo, studiamo il rapporto tra l'identità culturale di allievi emigrati d'origine portoghese che hanno terminato la scuola dell'obbligo in Svizzera (canton Vaud) e alcune loro caratteristiche personali, famigliari e scolastiche. L'identità culturale è misurata tramite un adattamento della scala d'acculturazione di Berry. I risultati mostrano che questi allievi tendono a voler conservare la loro cultura d'origine pur integrando la cultura svizzera (posizione pro-integrazione). D’altronde, essi sono contro un rifiuto della cultura svizzera (posizione anti-separazione) e portoghese (posizione anti-assimilazione). Costatiamo però delle di- 
minuzioni di queste posizioni, in particolare di quella pro-integrazione, in funzione di alcune caratteristiche personali (sesso, data d'arrivo in Svizzera), famigliari (tipo di permesso di lavoro della madre) e scolastiche (ripetizione dell'anno scolastico, livello di riuscita scolastica, frequentazione dei corsi di lingua e di civilizzazione portoghese).

\section{Cultural identity and school characteristics of Portuguese pupils in Switzerland}

\section{Summary}

In this article, we study the relationship between the cultural identity and certain personal, familial and school characteristics of Portuguese pupils finishing their obligatory school in Switzerland (canton of Vaud). The cultural identity is assessed with an adaptation of Berry's acculturation scale. The results show that the pupils have a tendency to very clearly preserve their Portuguese culture, while integrating the Swiss culture in the same time (attitude clearly pro-integration). Moreover, they are against a rejection of the Swiss culture (attitude clearly antiseparation) or of the Portuguese culture (attitude slightly anti-assimilation). However, in function of certain personal (sex, date of arrival in Switzerland), familial (type of mother's work permit), and school (resetting of years, general level of school success, attendence of the civilisation and language Portuguese courses) characteristics, there is a significant bending of certain of this attitudes and in particular of the pro-integration attitude. These results are discussed from the point of view of both their school and political implications (danger of school and social marginalisation). 